\title{
Arbitrage-free multifactor term structure models: a theory based on stochastic control
}

\author{
Andrea Gombani \\ ISIB-CNR * \\ Wolfgang J. Runggaldier \\ Dipartimento di Matematica Pura ed Applicata \\ Università di Padova ${ }^{\dagger}$
}

\begin{abstract}
We present an alternative approach to the pricing of bonds and bond derivatives in a multivariate factor model for the term structure of interest rates that is based on the solution of an optimal stochastic control problem. It can also be seen as an alternative to the classical approach of computing forward prices by forward measures and as such can be extended to other situations where traditionally a change of measure is involved based on a change of numeraire. We finally provide explicit formulas for the computation of bond options in a bivariate linear-quadratic factor model.
\end{abstract}

Key words: Multi-factor term structures, bond option pricing, stochastic control.

AMS subject classification: 91B28, 93E20.

\section{Introduction}

The use of the Girsanov transformation to obtain a martingale measure has become a fundamental tool of asset and bond pricing. The key feature of this technique is a change of drift which preserves trajectories. However, as it is well known, this is not the only

*Corso Stati Uniti 4, 35127 Padova, Italy, e-mail: gombani@isib.cnr.it, corresponding author

†Via Trieste 63, 35121 Padova, e-mail: runggal@math.unipd.it 
way to change the drift of a stochastic process. In fact, the drift can also be changed by feedback, albeit with a change of the trajectories, but keeping the same measure.

The main purpose of this paper is to show that, for a diffusion-type multifactor model with factor process $x_{t}$, a feedback approach resulting from a stochastic control methodology provides the same pricing model for bonds and bond derivatives as that obtained by the traditional martingale approach, but without changing the measure at all. This equivalence is shown to take a particularly appealing form in the case of a linear-quadratic factor model for the term structure (a term structure where the bond prices are exponentially quadratic in the factors with the latter satisfying linear-Gaussian dynamics). In this latter case the control problem becomes in fact of the classical linear-quadratic type.

The major novelty of our approach can thus be seen in the linking of stochastic optimal control theory with the classical martingale approach thereby providing an alternative representation of prices and forward prices of bonds under a multi-factor term structure.

Stochastic control techniques have been adopted quite early in finance, but mainly for portfolio optimization. Starting from Merton [1971] many papers have appeared dealing with various aspects of this problem (risk sensitive dynamic asset management, beating a moving target, problems with transaction costs and constraints, etc.), various methodologies (Hamilton-Jacobi-Bellman approach of Dynamic programming, martingale method and convex duality, impulse control, backwards stochastic differential equations) and various criteria (mean variance, expected utility, almost sure optimality, and down to the more recent behavioral approaches). Insurance is a related field, where stochastic control has also been fruitfully applied mainly for the purpose of minimizing ruin probability. To the best of our knowledge, stochastic control has however not yet been applied in the context of derivative pricing and our purpose here is to present an application to pricing in multifactor term structure models.

As already mentioned, the pivotal aspect of our approach lies in the change of drift: while in the traditional martingale approach a change of drift is implicit in the change of measure, in the control approach the drift is changed, without changing the measure, by applying a control that turns out to be in feedback form, namely as a function of the state/factor process and this leads to a so-called closed-loop model. The trajectories of the factors in a closed-loop model are changed with respect to those of the corresponding open loop model, but for the bond pricing this is quite irrelevant. In fact, since the observed values are eventually the rates and bond prices, it is quite indifferent, as far as pricing is concerned, whether these values are generated by an original open loop model with different trajectories (which we never observe) and the same measure, or same trajectories and a different measure. What is relevant is that they produce the same prices.

The immediate implication of our approach is, in our view, the novel insight that it is equivalent to calculate prices either on the basis of a traditional change of measure or by solving an optimal stochastic control problem. This equivalence is worked out in detail for prices and forward prices of bonds and, more generally, for forward measures with application to bond option pricing. It can furthermore be extended to other analogous 
situations such as swap measures for the pricing of interest rate swaps.

In addition to its theoretical interest, the equivalence also allows for the use of wellknown control theory tools to obtain computational pricing algorithms and, in fact, straightforward expressions are derived to calculate the prices of options on bonds in the linear-quadratic case: in particular, when the factor process is one- or two-dimensional, these computation are amenable to those of simple line integrals.

The paper is structured as follows. In section 2 we introduce the basic setup for the general nonlinear case as well as for the specific linear-quadratic factor model. Section 3 deals with prices and forward prices of bonds, presented first for the general nonlinear case (subsections 3.1 and 3.3) and then for the specific linear-quadratic case (subsections 3.2 and 3.2.1 as well as 3.3.1). The extension to forward measures and application to bond option pricing is the subject of Section 4 where, for the specific linear-quadratic case, we also provide explicit bond option pricing formulas. The last section 5 is devoted to concluding remarks and possible extensions. An Appendix contains some specific technical aspects.

\section{Arbitrage free derivation for the term structure}

On a filtered probability space $\left(\Omega, \mathcal{F}, \mathcal{F}_{t}, Q\right)$, where expectation under $Q$ will in the sequel simply be denoted by $E$, consider a factor process satisfying

$$
d x(t)=\mathbf{f}(t, x(t)) d t+\mathbf{g}(t, x(t)) d w(t) \quad, \quad 0 \leq t \quad x(0)=0
$$

where $x(t)$ has dimension $n, w$ is a $k$-dimensional Wiener process w.r. to $\left(Q, \mathcal{F}_{t}\right)$. We denote the bond prices by $p(t, T, x)$, where $t$ is the time variable, $T$ is the maturity of the bond and $x$ is the value of the factor process $x$, evaluated at time $t$; this notation is being adopted to stress the fact that the bond price depends also on the factor process (2.1). Analogously the forward rate corresponding to $p(t, T, x)$ will be denoted as $f(t, T, x):=-\frac{\partial}{\partial T} \ln p(t, T, x)$ and for the short rate we have $r(t, x):=f(t, t, x)$. When appropriate, for the latter we shall also write $r(t, x(t))$ (we shall use the shorthand notations $p(t, T)$ and $r(t)$ only when the meaning is clear). We shall make the following

Assumption 2.1 There exists $M>0$ such that

$$
\|\mathbf{f}(t, x)\| \leq M(1+\|x\|),\|\mathbf{g}(t, x)\| \leq M,|r(t, x)| \leq M\left(1+\|x\|^{2}\right)
$$

We recall now the Term Structure Equation (see e.g. Björk, [2004]),

Proposition 2.2 Assume $p(t, T, x)$ to be of class $\mathcal{C}^{1,2}$. A sufficient condition for the term structure induced by $p(t, T, x)$ to be arbitrage-free is that $p(t, T, x)$ satisfies the following 
partial differential equation

$$
\left\{\begin{array}{l}
\frac{\partial}{\partial t} p(t, T, x)+\mathbf{f}^{\prime}(t, x) \nabla_{x} p(t, T, x)+\frac{1}{2} \operatorname{tr}\left(\mathbf{g}^{\prime}(t, x) \nabla_{x x} p(t, T, x) \mathbf{g}(t, x)\right) \\
\quad-p(t, T, x) r(t, x)=0 \\
p(T, T, x)=1
\end{array}\right.
$$

Remark 2.3 Notice that, under Assumption 2.1, equation (2.1) has a unique strong solution. Furthemore, if $p(t, T, x)$ satisfies equation (2.2) then, under Assumption 2.1, it is the unique solution of (2.2) within the class of functions satisfying the growth condition $|p(t, T, x)| \leq C e^{C\|x\|^{2}}$ for all $t \leq T$ and all $x \in \mathbb{R}^{n}$, where $C$ is some positive constant, possibly depending on $T$ (see e.g. Ch. 6, §4 in Friedman [1975]).

Motivated by the classical affine term structure models, we shall consider below as particular case an exponentially quadratic output model as an instance of a non-affine model. This case results from particularizing (2.1) to linear dynamics and by modeling the short rate as a quadratic function of the factors. By imposing the conditions of absence of arbitrage, it can be shown (see Filipovic [2001]) that, for a linear-Gaussian factor model, general exponentially polynomial output models with degree larger than 2 reduce to the quadratic output model, i.e. the coefficients of the powers of $x$ larger than 2 have to be equal to zero. The linear factor - nonlinear output model will be used below to obtain specific results. Dually one could also consider nonlinear factor - affine output models and there is also some equivalence between the two possible settings. In Subsection 4.2 we will show that in case of a linear factor - quadratic output model explicit computations involving just numerical integration of line integrals can be carried out. Various popular models (Vasiček, Cox-Ingersoll-Ross, Ho-Lee, Hull-White) are amenable to this setting.

\subsection{Linear Dynamics (exponential quadratic term structure)}

We make the convention that all vectors (including the gradient) will be column vectors. Transposition will be denoted by a prime (').

It is not restrictive, in practice, to assume that there exists a largest maturity of interest $T_{0}>0$ such that $T \leq T_{0}$ for all maturities $T$. We have the following result:

Proposition 2.4 Let $x(t)$ be as in (2.1)

$$
\left\{\begin{array}{l}
\mathbf{f}(t, x)=F(t) x+H(t) \\
\mathbf{g}(t, x)=G(t) \quad(\text { independent of } x) \\
r(t, x)=a(t)+x^{\prime} b(t)+x^{\prime} c(t) x
\end{array}\right.
$$

where $a(t)$ is a scalar quantity, $H(t), b(t)$ are $n$-dimensional column vectors and $F(t), c(t)$ are matrices of dimension $n \times n$ with $c(t) \geq 0$ and symmetric and $G(t)$ is of dimension $n \times$ 
$k$. These functions are supposed to be continuous in $t$ on the interval $\left[0, T_{0}\right]$. Furthermore, let $C(t, T), B(t, T), A(t, T)$ satisfy the following system of ODEs

$$
\begin{gathered}
\begin{aligned}
& \frac{\partial}{\partial t} C(t, T)+2 F(t) C(t, T)-2 C(t, T) G(t) G^{\prime}(t) C(t, T)+c(t)=0 \\
& \frac{\partial}{\partial t} B(t, T)+ F^{\prime}(t) B(t, T)+2 C(t, T) H(t)-2 G^{\prime}(t) G(t) C(t, T) B(t, T) \\
&+b(t)=0 \\
& \frac{\partial}{\partial t} A(t, T)+ B^{\prime}(t, T) H(t)-\frac{1}{2} B^{\prime}(t, T) G(t) G^{\prime}(t) B(t, T) \\
&+\operatorname{tr}\left(G^{\prime}(t) C(t, T) G(t)\right)+a(t)=0, \\
& C(T, T)=0 \quad B(T, T)=0 \quad A(T, T)=0
\end{aligned}
\end{gathered}
$$

Then

$$
p(t, T, x)=\exp \left[-A(t, T)-x^{\prime} B(t, T)-x^{\prime} C(t, T) x\right]
$$

defines an arbitrage-free term structure.

Proof: By (2.3) and the hypotheses of the Proposition, Assumption 2.1 is satisfied and so the solution to (2.2) is unique (see Remark 2.3). Suppose that it is indeed of the form (2.7). Then, denoting by a subscript the partial derivative with respect to $t$,

$$
\begin{aligned}
p_{t}(t, T, x)= & p(t, T, x)\left[-A_{t}(t, T)-x^{\prime} B_{t}(t, T)-x^{\prime} C_{t}(t, T) x\right] \\
\nabla_{x} p(t, T, x)= & p(t, T, x)[-B(t, T)-2 C(t, T) x] \\
\nabla_{x x} p(t, T, x)= & p(t, T, x)\left[B(t, T) B^{\prime}(t, T)+4 C(t, T) x x^{\prime} C(t, T)\right. \\
& \left.+2 B(t, T) x^{\prime} C(t, T)+2 C(t, T) x B^{\prime}(t, T)-2 C(t, T)\right]
\end{aligned}
$$

Substituting these expressions into (2.2) and dividing by $p(t, T, x)$ we obtain

$$
\begin{aligned}
& -A_{t}(\cdot)-x^{\prime} B_{t}(\cdot)-x^{\prime} C_{t}(\cdot) x-H^{\prime} B(\cdot)-x^{\prime} F^{\prime} B(\cdot) \\
& -2 H^{\prime} C(\cdot) x-2 x^{\prime} F^{\prime} C(\cdot) x+\frac{1}{2} B^{\prime}(\cdot) G(t) G^{\prime}(t) B(\cdot)+2 x^{\prime} C(\cdot) G(t) G^{\prime}(t) C(\cdot) x \\
& +2 \operatorname{tr}\left(G^{\prime}(t) B(\cdot) x^{\prime} C(\cdot) G(t)\right)-\operatorname{tr}\left(G^{\prime}(t) C(\cdot) G(t)\right)-a(t)-x^{\prime} b(t)-x^{\prime} c(t) x=0
\end{aligned}
$$

Since this latter relation has to hold for all possible values of $x$ that correspond to a solution of (2.1), we set the second order, first order and constant terms equal to zero thereby obtaining the desired result with the boundary conditions following from $p(T, T, x)=1, \forall x$.

While the above condition ensures that the discounted price processes $p(t, T)$ are martingales, the initial condition, that is the observed bond prices $p^{*}(0, T)$ at the time $t=0$, is not necessarily matched. This is easily obtained, though, by imposing a condition 
on $a(t)$. To this end, in accordance with the definition of forward rates, we put for the observed forward rates

$$
f^{*}(0, T):=-\frac{\partial}{\partial T} \log p(0, T)
$$

Lemma 2.5 Let the term structure $p(t, T)$ satisfy the conditions of Proposition 2.4 and let the observed prices $p^{*}(0, T)$ (and the associated forward rates $f^{*}(0, T)$ be given). Then

$$
p(0, T)=p^{*}(0, T)
$$

if and only if the deterministic coefficient $a(t)$ of $r(t, x)$ in (2.3) satisfies

$$
a(t):=f^{*}(0, t)+\int_{0}^{t} \beta(s, t) d s
$$

having put

$$
\begin{aligned}
\beta(t, T):=-\frac{\partial}{\partial T} & \left(-B^{\prime}(t, T) H(t)+\frac{1}{2} B^{\prime}(t, T) G(t) G^{\prime}(t) B(t, T)\right. \\
& -\operatorname{tr}\left(G^{\prime}(t) C(t, T) G(t)\right)
\end{aligned}
$$

Proof: Since we assume $x(0)=0$, from (2.7) we get $p(0, T, x)=\exp \{-A(0, T)\}$, and thus we can integrate (2.6) with initial condition $A(0, T)=\int_{0}^{T} f^{*}(0, s) d s$ to obtain:

$$
\begin{aligned}
A(t, T)=-\int_{0}^{t}\left(B^{\prime}(s, T) H(s)-\frac{1}{2} B^{\prime}(s, T) G(s) G^{\prime}(s) B(s, T)\right. & \\
& \left.\quad+\operatorname{tr}\left(G^{\prime}(s) C(s, T) G(s)\right)+a(s)\right) d s+\int_{0}^{T} f^{*}(0, s) d s
\end{aligned}
$$

Since $A(T, T)=0$ for all $T$ (as well as $B(T, T)=0$ and $C(T, T)=0$ ), differentiation of the above expression in $t=T$ yields

$$
\begin{aligned}
0= & -\frac{\partial}{\partial T} \int_{0}^{T}\left(B^{\prime}(s, T) H(s)-\frac{1}{2} B^{\prime}(s, T) G(s) G^{\prime}(s) B(s, T)\right. \\
& \left.\quad+\operatorname{tr}\left(G^{\prime}(s) C(s, T) G(s)\right)+a(s)\right) d s+f^{*}(0, T) \\
= & \int_{0}^{T} \beta(s, T) d s-a(T)+f^{*}(0, T)
\end{aligned}
$$

which is, in fact, (2.9). 


\section{$3 \quad$ Prices and forward prices of bonds}

Here we want to show how prices and forward prices of bonds can be related to the solution of a suitable stochastic control problem. To this effect we have to associate to the Term Structure Equation (2.2) a stochastic control problem and this can be achieved in two steps:

i) Apply a convex transformation to the bond price and obtain from the Term Structure Equation a (nonlinear) PDE satisfied by the transformed function.

ii) Rewrite the nonlinear PDE in the form of a HJB equation and determine the corresponding stochastic control problem.

\subsection{Bond pricing as a stochastic control problem}

Inspired by a result in Fleming [1982] we apply more specifically a logarithmic transform as an instance of a convex transform to $p(t, T, x)$, namely

$$
W(t, T, x):=-\log p(t, T, x)
$$

and obtain from (2.2) the following PDE after division by $e^{-W(t, T, x)}$

$$
\frac{\partial}{\partial t} W+\mathbf{f}^{\prime} \nabla_{x} W-\frac{1}{2}\left(\nabla_{x} W\right)^{\prime} \mathbf{g g}^{\prime} \nabla_{x} W+\frac{1}{2} \operatorname{tr}\left\{\mathbf{g}^{\prime} \nabla_{x x} W \cdot \mathbf{g}\right\}+r=0
$$

with terminal condition $W(T, T, x) \equiv 0$. Consider now the Hamilton-Jacobi-Bellman (HJB) equation:

$$
\frac{\partial}{\partial t} W+\inf _{u \in \mathbb{R}^{k}}\left\{\left(\mathbf{f}^{\prime}+u^{\prime} \mathbf{g}^{\prime}\right) \cdot \nabla_{x} W+\frac{1}{2} \operatorname{tr}\left(\mathbf{g}^{\prime} \nabla_{x x} W \mathbf{g}\right)+\frac{1}{2} u^{\prime} u+r\right\}=0
$$

that has as solution

$$
u(t, x):=-\mathbf{g}^{\prime}(t, x) \nabla_{x} W(t, x)
$$

and notice that, by substituting this solution into (3.3), this latter equation becomes (3.2).

Definition 3.1 In what follows we shall call admissible a control in feedback form, i.e. such that $u(t)=u(t, x(t))$, for which in (3.5) below the equation for $x(t)$ has a unique weak solution and the expectation there is finite. We shall denote by $\mathcal{U}$ the class of admissible controls.

Notice now that $W(t, T, x)$ can be seen as solution to the HJB equation (3.3) associated to the following stochastic control problem

$$
\left\{\begin{array}{l}
d x(t)=[\mathbf{f}(t, x(t))+\mathbf{g}(t, x(t)) u(t)] d t+\mathbf{g}(t, x(t)) d w(t) \\
W(t, T, x)=\min _{u \in \mathcal{U}} E_{t, x}\left\{\int_{t}^{T}\left[\frac{1}{2} u^{\prime}(s) u(s)+r(s)\right] d s\right\}
\end{array}\right.
$$


and we also have

$$
p(t, T, x)=e^{-W(t, T, x)}
$$

The relation (3.6) can be seen as a control or a "variational" interpretation of the bond prices.

Remark 3.2 The present formulation reflects somehow the point of view of the issuer of the bond: in fact, the problem can be seen as minimizing the yields of the bond, subject to the constraint of a given dynamics in the first line of (3.5) and with a quadratic penalty function for the control. There seems to be no apparent problem formulation reflecting the point of view of the buyer of the bond.

Remark 3.3 The terminal condition $W(T, T, x)=0$ arises from the fact that we are pricing bonds. A more general result can be derived, which allows for the computation of option prices. This will be done in the next section for linear dynamics as specified in (2.3). Notice that, in principle, the quadratic function $r(t, x)$ in (3.2) can have arbitrary coefficients. Nevertheless, if we want the bond prices to match those induced by a particular observed forward rates curve $f^{*}(0, T)$, the coefficient a $(t)$ will have to satisfy (2.9).

\subsection{The case of linear dynamics (exponential quadratic term structure)}

Consider now the particular case where the dynamics in (2.1) is linear, the short rate is a quadratic function of the factors and a quadratic terminal condition is imposed on $W(\cdot)$. Due to (2.3) the first equation in (3.5) then reduces to

$$
d x(t)=[F(t) x(t)+H(t)+G(t) u(t)] d t+G(t) d w(t)
$$

The expression in (2.3) for the rates $r(t, x)$ then yields immediately the following expression for $W(t, T, x)$ in (3.5) (the order of the terms is changed for better emphasis)

$$
\begin{gathered}
W(t, T, x):=\min _{u \in \mathcal{U}} E_{t, x}\left\{\int_{t}^{T}\left(x^{\prime}(s) c(s) x(s)+x^{\prime}(s) b(s)+a(s)+\frac{1}{2} u^{\prime}(s) u(s)\right) d s\right. \\
\left.+x^{\prime}(T) C^{T} x(T)+x^{\prime}(T) B^{T}+A^{T}\right\}
\end{gathered}
$$

where $c(t), b(t), a(t), C^{T}, B^{T}, A^{T}$ are given functions/constants with $c(t) \geq 0$ and $C^{T} \geq 0$ and both symmetric.

The Hamilton-Jacobi-Bellman (HJB) equation (3.3) for $W(t, T, x)$ becomes

$$
\left\{\begin{array}{c}
\frac{\partial W}{\partial t}(t, T, x)+\inf _{u \in \mathbb{R}^{k}}\left\{x^{\prime} c(t) x+x^{\prime} b(t)+a(t)+\frac{1}{2} u^{\prime} u\right. \\
\quad+\left[x^{\prime} F^{\prime}(t)+H^{\prime}(t)+u^{\prime} G^{\prime}(t)\right] \nabla_{x} W(t, T, x) \\
\left.\quad+\frac{1}{2} \operatorname{tr}\left(G^{\prime}(t) \nabla_{x x} W(t, T, x) G(t)\right)\right\}=0 \\
W(T, T, x)=\left(x^{\prime} C^{T} x+x^{\prime} B^{T}+A^{T}\right)
\end{array}\right.
$$


We have

Proposition 3.4 The minimizing $u$ in (3.9) is

$$
u^{*}(t, x ; W)=-G^{\prime}(t)\left(\nabla_{x} W(t, T, x)\right)
$$

and the solution $W(t, T, x)$ is given by

$$
W(t, T, x)=x^{\prime} C(t, T) x+x^{\prime} B(t, T)+A(t, T)
$$

where $C(t, T), B(t, T), A(t, T)$ satisfy the following equations and terminal conditions at $t=T$

$$
\left\{\begin{aligned}
\frac{\partial}{\partial t} C(t, T)+ & F^{\prime}(t) C(t, T)+C(t, T) F(t) \\
& -2 C(t, T) G(t) G^{\prime}(t) C(t, T)+c(t)=0, \\
\frac{\partial}{\partial t} B(t, T)+ & F^{\prime}(t) B(t, T)+2 C(t, T) H(t) \\
& -2 C(t, T) G(t) G^{\prime}(t) B(t, T)+b(t)=0, \\
\frac{\partial}{\partial t} A(t, T)+ & H^{\prime}(t) B(t, T)+\operatorname{tr}\left(G^{\prime}(t) C(t, T) G(t)\right) \\
& -\frac{1}{2} B^{\prime}(t, T) G(t) G^{\prime}(t) B(t, T)+a(t)=0, \\
C(T, T)= & C^{T}, B(T, T)=B^{T}, A(T, T)=A^{T}
\end{aligned}\right.
$$

with the values $A^{T}, B^{T}, C^{T}$ of the terminal conditions corresponding to those of the terminal condition in (3.9).

Notice that, since $p(t, T, x)=e^{-W(t, T, x)}$, in this case of linear dynamics we obtain an exponential quadratic term structure.

Proof: Notice that, if $B(t, T) \equiv 0$, then the above reduces to the familiar LQG problem (see e.g. Fleming et al. [1975], Øksendal [1998], see also chapter 19 in Björk, [2004]). The problem is amenable to the classical LQG problem whenever it is possible to complete the squares. This is the case if $c$ is full-rank or more generally if $b(t) \in \operatorname{Img} c(t)$ for $0 \leq t \leq T$. If these conditions are not satisfied, though, the function $r(t, x)$ is not strictly convex in $x$, but the problem still admits a unique solution. The proof is quite straightforward. We refer to the Appendix A for details.

Remark 3.5 The interesting fact here is that (3.12) corresponds to (2.4)-(2.6) even if (3.12) is slightly more general than (2.4)-(2.6): there we have $A^{T}=0, B^{T}=0, C^{T}=0$. The general formulation here will be needed to accommodate forward prices (see Section 3.3). In the next subsection we shall consider in more detail the specific arbitrage free Term Structure derivation so as to match the observed rates $f^{*}(0, T)$. Concerning the actual solution of $C(t, T)$ and $B(t, T)$ in (3.12) see Appendix $B$. 
Remark 3.6 From a financial point of view it could be of interest to consider also the case of a linear factor model with adapted coefficients. Such a model is however not Markovian and so does not allow to use our PDE-based approach. On the other hand in the literature one can find approaches to solve a linear-quadratic control problem with adapted coefficients. To this effect see Hu et al. [2005], where the authors use a direct approach that does not involve HJB equations.

\subsubsection{Matching the observed rates}

As pointed out in the second part of Section 2.1, to match an observed forward rates curve $f^{*}(0, T)$, conditions on the the deterministic part $a(t)$ of the rate process $r(t, x)$ in (2.3) have to be imposed. In view of Proposition 3.4, this can be achieved in the present linear framework by setting $A^{T}=0, B^{T}=0, C^{T}=0$ and using Lemma 2.5. We have in fact the following result

Theorem 3.7 Let $r(t)$ be defined as in (2.3) and let the bond price $p(t, T, x)$ be given by

$$
p(t, T, x)=E_{t, x} e^{\left\{-\int_{t}^{T} r(s) d s\right\}}
$$

Then

$$
p(t, T, x)=e^{-W(t, T, x)}
$$

where $W$ is, for each $T$, of the form (3.11)-(3.12) with $A^{T}=B^{T}=C^{T}=0$ and a(t) in (3.12) is determined by the boundary condition

$$
W(0, T, 0)=\int_{0}^{T} f^{*}(0, s) d s
$$

where $f^{*}(0, t)$ is the initially observed forward rate.

Proof: In view of Proposition 3.4 with $A^{T}=0, B^{T}=0, C^{T}=0, W(t, T, x)$ satisfies (3.9) and thus $p(t, T, x)=e^{-W(t, T, x)}$ satisfies (2.2). The only thing to show then is that $a(t)$ is of the form (2.9). But this can be obtained by using the same argument as in Lemma 2.5.

Note that the function $a(t)$ in (2.9) is expressed as the integral of a partial derivative. Since the computation of $A(t, T)$ requires another integration, this expression might seem quite cumbersome. Nevertheless, we now show that $A(t, T)$ allows for the following 
representation, whose computation requires a simple line integral:

$$
\begin{aligned}
& A(t, T)=\int_{t}^{T} H^{\prime}(s) B(s, T) d s+\int_{t}^{T}\left[\operatorname{tr}\left(G^{\prime}(s) C(s, T) G(s)\right)\right. \\
& \left.-\frac{1}{2} B(s, T) G(s) G^{\prime}(s) B^{\prime}(s, T)\right] d s+\int_{t}^{T} a(s) d s
\end{aligned}
$$

$$
\begin{aligned}
= & \int_{t}^{T} H^{\prime}(s) B(s, T) d s+\int_{t}^{T}\left[\operatorname{tr}\left(G^{\prime}(s) C(s, T) G(s)\right)-\frac{1}{2} B(s, T) G(s) G^{\prime}(s) B^{\prime}(s, T)\right] d s+ \\
& \int_{0}^{T} a(s) d s-\int_{0}^{t} a(s) d s
\end{aligned}
$$

$=\int_{t}^{T} H^{\prime}(s) B(s, T) d s+\int_{t}^{T}\left[\operatorname{tr}\left(G^{\prime}(s) C(s, T) G(s)\right)-\frac{1}{2} B(s, T) G(s) G^{\prime}(s) B^{\prime}(s, T)\right] d s$

$-\int_{0}^{T}\left[\operatorname{tr}\left(G^{\prime}(s) C(s, T) G(s)\right)-\frac{1}{2} B(s, T) G(s) G^{\prime}(s) B^{\prime}(s, T)\right] d s+\int_{0}^{T} f^{*}(0, s) d s$

$+\int_{0}^{t}\left[\operatorname{tr}\left(G^{\prime}(s) C(s, t) G(s)\right)-\frac{1}{2} B(s, t) G(s) G^{\prime}(s) B^{\prime}(s, t)\right] d s-\int_{0}^{t} f^{*}(0, s) d s$

$$
\begin{gathered}
=\int_{t}^{T} f^{*}(0, s) d s+\int_{t}^{T} H^{\prime}(s) B(s, T) d s-\int_{0}^{t} \operatorname{tr}\left[G^{\prime}(s) C(s, T) G(s)-G^{\prime}(s) C(s, t) G(s)\right] d s \\
+\frac{1}{2} \int_{0}^{t}\left[B^{\prime}(s, T) G(s) G^{\prime}(s) B(s, T)-B^{\prime}(s, t) G(s) G^{\prime}(s) B(s, t)\right] d s
\end{gathered}
$$

\subsection{Forward prices}

We compute now the forward price of a bond, that is the value $E_{t, x}^{Q_{\tau}} p(\tau, T, x)$ for a given $\tau$ with $t \leq \tau \leq T$, and where $Q_{\tau}$ is the forward measure with respect to the numeraire $p(t, \tau, x)$. Since prices expressed in units of $p(t, \tau, x)$ are $Q_{\tau}$-martingales, we have

$$
E_{t, x}^{Q_{\tau}} p(\tau, T, x)=\frac{p(t, T, x)}{p(t, \tau, x)}
$$

Recall that in $p(t, \tau, x)$ the dependence on the factor process $x$ is through its value at time $t$.

The key ingredient to compute prices in the previous section 3.2 was the change of the dynamics by feedback: in fact, for each $t$ and $T$ the closed loop dynamics

$$
d x^{T}(t)=\left[\left(\mathbf{f}\left(t, x^{T}(t)\right)-\mathbf{g}\left(t, x^{T}(t)\right) \mathbf{g}^{\prime}\left(t, x^{T}(t)\right) \nabla_{x} W\left(t, T, x^{\tau}(t)\right)\right] d t+\mathbf{g}\left(t, x^{T}(t)\right) d w(t)\right.
$$

was derived by substituting (3.4) into (3.5). The dynamics (3.5), in turn, was obtained by adding a control term to the original dynamics (2.1). Computing the differential of 
$p\left(t, T, x^{T}\right)$ along the trajectories of (3.17) yields (using (3.2))

$$
\begin{aligned}
d p\left(t, T, x^{T}\right)= & d e^{-W\left(t, T, x^{T}\right)}= \\
= & -p\left(t, T, x^{T}\right)\left\{\frac{\partial}{\partial t} W\left(t, T, x^{T}\right)+\left[\mathbf{f}^{\prime}\left(t, x^{T}\right)\right.\right. \\
& \left.-\left(\nabla_{x} W\right)^{\prime}\left(t, T, x^{T}\right) \mathbf{g}\left(t, x^{T}\right) \mathbf{g}^{\prime}\left(t, x^{T}\right)\right] \nabla_{x} W\left(t, T, x^{T}\right) \\
(3.18)= & -\frac{1}{2}\left(\nabla_{x} W\right)^{\prime}\left(t, T, x^{T}\right) \mathbf{g}\left(t, x^{T}\right) \mathbf{g}^{\prime}\left(t, x^{T}\right) \nabla_{x} W\left(t, T, x^{T}\right) \\
& \left.+\frac{1}{2} \operatorname{tr}\left\{\mathbf{g}^{\prime}\left(t, x^{T}\right) \nabla_{x x} W\left(t, T, x^{T}\right) \cdot \mathbf{g}\left(t, x^{T}\right)\right] d t+\left(\nabla_{x} W\right)^{\prime}\left(t, T, x^{T}\right) \mathbf{g}\left(t, x^{T}\right) d w(t)\right\} \\
= & p\left(t, T, x^{T}\right)\left\{\left[\left(\nabla_{x} W\right)^{\prime}\left(t, T, x^{T}\right) \mathbf{g}\left(t, x^{T}\right) \mathbf{g}^{\prime}\left(t, x^{T}\right) \nabla_{x} W\left(t, T, x^{T}\right)\right.\right. \\
& \left.+r(t)] d t-\left(\nabla_{x} W\right)^{\prime}\left(t, T, x^{T}\right) \mathbf{g}\left(t, x^{T}\right) d w(t)\right\}
\end{aligned}
$$

To get some intuition of why the dynamics (3.17) is of interest, observe the following fact: it can be shown (see e.g. Björk, [2004] and also formula (4.2) below) that the Girsanov kernel from $Q$ to $Q_{T}$ is $-\left(\nabla_{x} W\right)^{\prime}\left(t, T, x^{T}\right) \mathbf{g}\left(t, x^{T}\right)$ and thus the dynamics of $p\left(t, T, x^{T}\right)$ in (3.18) is exactly the one corresponding to the forward measure with numeraire $p(t, T, x)$. The formal equivalence will be proved in Section 4.1. Now this is also true for all $\tau<T$ and therefore, in order to express the price processes (3.16) in terms of a control problem, it seems natural to try and use, instead of (2.1), the following dynamics $x^{\tau}(t)$ in the measure $Q$

$$
d x^{\tau}(t)=\left[\left(\mathbf{f}\left(t, x^{\tau}(t)\right)-\mathbf{g}\left(t, x^{\tau}(t)\right) \mathbf{g}^{\prime}\left(t, x^{\tau}(t)\right) \nabla_{x} W\left(t, \tau, x^{\tau}(t)\right)\right] d t+\mathbf{g}\left(t, x^{\tau}(t)\right) d w(t)\right.
$$

where $W(t, \tau, x)$ is as in (3.1) with $T=\tau$ and satisfies (3.2) with terminal condition $W(\tau, \tau, x)=0$; furthermore, $x^{\tau}(\cdot)$ has the same initial condition as $x(\cdot)$. We now show that this is the right construction (see also Section 4.1 below). For the just stated purpose introduce the following quantity

$$
p^{\tau}(t, T, x):=E_{t, x} p(\tau, T, x)=E_{t, x}\left\{\exp \left[-W\left(\tau, T, x^{\tau}\right)\right]\right\} \quad t \leq \tau \leq T
$$

(since $x(\cdot)$ and $x^{\tau}(\cdot)$ both have the same initial condition $x$, we shall use the notation $p^{\tau}(t, T, x)$ when the factor process is $x^{\tau}$ starting in $x$ at $\left.t\right)$. Notice that the second equality follows from (3.6). The Kolmogorov backward equation to compute this expected value is (dropping the variables):

$$
\left\{\begin{array}{l}
\frac{\partial}{\partial t} p^{\tau}+\left[\mathbf{f}^{\prime}-\left(\nabla_{x} W\right)^{\prime} \mathbf{g g}^{\prime}\right] \nabla_{x} p^{\tau}+\frac{1}{2} \operatorname{tr}\left(\mathbf{g}^{\prime} \nabla_{x x} p^{\tau} \mathbf{g}\right)=0 \\
p^{\tau}(\tau, T, x)=\exp [-W(\tau, T, x)]
\end{array}\right.
$$

Remark 3.8 The derivation here and up to the next Proposition 3.9 parallels that in section 3.1 with equation (3.21) corresponding to (2.2). Assumption 2.1 guarantees uniqueness of the solution to (2.2) and this uniqueness will be used in the proof of the the next 
Proposition 3.9. The same assumption 2.1 is however not sufficient to guarantee uniqueness also of the solution to (3.21). For our purposes here uniqueness of (3.21) is however not strictly needed. By strengthening our assumptions and requiring $\nabla_{x} W(\cdot)$ to have at most linear growth in $x$, an assumption that is satisfied in the particular linear-quadratic case of the next subsection 3.3.1, we can obtain uniqueness here too.

Setting

$$
W^{\tau}(t, T, x):=-\ln p^{\tau}(t, T, x)
$$

(3.21) leads to

$$
\left\{\begin{array}{l}
\frac{\partial}{\partial t} W^{\tau}+\left[\mathbf{f}^{\prime}-\left(\nabla_{x} W\right)^{\prime} \mathbf{g g} \mathbf{g}^{\prime}\right] \nabla_{x} W^{\tau}-\frac{1}{2}\left(\nabla_{x} W^{\tau}\right)^{\prime} \cdot \mathbf{g g}^{\prime} \nabla_{x} W^{\tau} \\
\quad+\frac{1}{2} \operatorname{tr}\left(\mathbf{g}^{\prime} \nabla_{x x} W^{\tau} \mathbf{g}\right)=0 \\
W^{\tau}(\tau, T, x)=W(\tau, T, x)
\end{array}\right.
$$

This equation results again from the following Hamilton-Jacobi-Bellman equation:

$$
\left\{\begin{array}{l}
\frac{\partial}{\partial t} W^{\tau}+\min _{u \in \mathbb{R}^{k}}\left\{\left[\mathbf{f}^{\prime}-\left(\nabla_{x} W\right)^{\prime} \mathbf{g g}^{\prime}+u^{\prime} \mathbf{g}^{\prime}\right] \nabla_{x} W^{\tau}+\frac{1}{2} \operatorname{tr}\left(\mathbf{g}^{\prime} \nabla_{x x} W^{\tau} \mathbf{g}\right)+\frac{1}{2} u^{\prime} u\right\}=0 \\
W^{\tau}(\tau, T, x)=W(\tau, T, x)
\end{array}\right.
$$

by substituting $u$ with the minimizer

$$
u(t, x):=-\mathbf{g}(t, x)^{\prime} \nabla_{x} W^{\tau}(t, x)
$$

Again, (3.24) is a HJB equation, this time associated with the following stochastic control problem, where the underlying measure is $Q$ and the admissible controls in $\mathcal{U}$ are those for which the equation for $x^{\tau}(t)$ has a unique weak solution and the expectation is finite.

$$
\left\{\begin{array}{r}
d x^{\tau}(t)=\left[\mathbf{f}\left(t, x^{\tau}(t)\right)-\left(\mathbf{g g}^{\prime}\right)\left(t, x^{\tau}(t)\right) \nabla_{x} W\left(t, \tau, x^{\tau}(t)\right)+\mathbf{g}\left(t, x^{\tau}(t)\right) u(t)\right] d t \\
+\mathbf{g}\left(t, x^{\tau}(t)\right) d w(t) \\
W^{\tau}(t, T, x)=\inf _{u \in \mathcal{U}} E_{t, x}\left\{\int_{t}^{\tau} \frac{1}{2} u^{\prime}(s) u(s) d s+W\left(\tau, T, x^{\tau}(\tau)\right)\right\}
\end{array}\right.
$$

We now show that in this way we have achieved the purpose of this section, namely we have

Proposition 3.9 Suppose that Assumption 2.1 is satisfied. Then the following chain of equalities holds

$$
E_{t, x}^{Q_{\tau}} p(\tau, T, x)=p^{\tau}(t, T, x)=E_{t, x}\left\{\exp \left[-W\left(\tau, T, x^{\tau}\right)\right]\right\}=\exp \left[-W^{\tau}(t, T, x)\right]
$$


Proof: In view of (3.16), for the first equality it suffices to show that, for $t \leq \tau \leq T$,

$$
p^{\tau}(\tau, T, x)=\frac{p(t, T, x)}{p(t, \tau, x)}
$$

i.e., in view also of (3.22) and of (3.6)

$$
\exp \left[-W^{\tau}(t, T, x)\right]=\frac{\exp [-W(t, T, x)]}{\exp [-W(t, \tau, x)]}
$$

which in turn is equivalent to showing that

$$
W(t, \tau, x)+W^{\tau}(t, T, x)=W(t, T, x)
$$

For this purpose we adapt an argument from Cogo [2011] putting

$$
\tilde{W}(t, x):=W(t, \tau, x)+W^{\tau}(t, T, x)
$$

Equations (3.2) for $W(\cdot)$ and $(3.23)$ for $W^{\tau}(\cdot)$ then imply

$$
\begin{aligned}
& -\frac{\partial}{\partial t} \tilde{W}=-\frac{\partial}{\partial t} W-\frac{\partial}{\partial t} W^{\tau}=\mathbf{f}^{\prime} \nabla_{x} W-\frac{1}{2}\left(\nabla_{x} W\right)^{\prime} \mathbf{g g}^{\prime} \nabla_{x} W \\
& +\frac{1}{2} \operatorname{tr} \mathbf{g}^{\prime} \nabla_{x x} W \mathbf{g}+r+\mathbf{f}^{\prime} \nabla_{x} W^{\tau}-\left(\nabla_{x} W\right)^{\prime} \mathbf{g g}^{\prime} \nabla_{x} W^{\tau} \\
& -\frac{1}{2}\left(\nabla_{x} W^{\tau}\right)^{\prime} \mathbf{g g}^{\prime} \nabla_{x} W^{\tau}+\frac{1}{2} \operatorname{tr}\left(\mathbf{g}^{\prime} \nabla_{x x} W^{\tau} \mathbf{g}\right) \\
& =\mathbf{f}^{\prime}\left[\nabla_{x} W+\nabla_{x} W^{\tau}\right]+\frac{1}{2} \operatorname{tr}\left(\mathbf{g}^{\prime}\left[W+W^{\tau}\right] \mathbf{g}\right)+r \\
& \quad-\frac{1}{2} \nabla_{x}\left(W+W^{\tau}\right)^{\prime} \mathbf{g g} \mathbf{g}^{\prime}\left(\nabla_{x}\left(W+W^{\tau}\right)\right)^{\prime} \\
& =\mathbf{f}^{\prime} \nabla_{x} \tilde{W}+\frac{1}{2} \operatorname{tr}\left(\mathbf{g}^{\prime} \tilde{W} \mathbf{g}\right)+r-\frac{1}{2}\left(\nabla_{x} \tilde{W}\right)^{\prime} \mathbf{g g}^{\prime} \nabla_{x} \tilde{W}
\end{aligned}
$$

with boundary condition

$$
\tilde{W}(\tau, x)=W(\tau, \tau, x)+W^{\tau}(\tau, T, x)=0+W(\tau, T, x)=W(\tau, T, x)
$$

It follows that $\tilde{W}(t, x)$ satisfies the same equation as $W(t, T, x)$, namely (3.2) with the same value at $t=\tau$. Now (3.2) is the equation satisfied by the one-to-one transformation (3.1) of the solution $p(t, T, x)$ of (2.2). Since, under our assumptions, the latter has a unique solution, so has the former and therefore

$$
\tilde{W}(t, x)=W(t, \tau, x)+W^{\tau}(t, T, x)=W(t, T, x) \quad \text { for } \quad t \leq \tau
$$

which proves (3.28) and thus the first equality in (3.27). The second equality follows from (3.20) and the third one from (3.22). 


\subsubsection{The case of linear dynamics (exponential quadratic term structure)}

Consider now again the particular case of linear dynamics (2.3) with the exponential quadratic term structure as in Section 3.2. Recall that for this case from (3.11) we have

$$
W(t, T, x)=-\log p(t, T, x)=A(t, T)+x^{\prime} B(t, T)+x^{\prime} C(t, T) x
$$

with $A(t, T), B(t, T), C(t, T)$ satisfying (3.12). The equation for $x^{\tau}(\cdot)$ in (3.19) then becomes

$$
d x^{\tau}(t)=\left[\left(F(t)-2 G(t) G^{\prime}(t) C(t, \tau)\right) x^{\tau}(t)+H(t)-G(t) G^{\prime}(t) B(t, \tau)\right] d t+G(t) d w(t)
$$

The relation (3.20) particularizes to

$$
\begin{aligned}
p^{\tau}(t, T, x) & :=E_{t, x} p(\tau, T, x) \\
& =E_{t, x}\left\{\exp \left[-\left(x^{\tau}(\tau)\right)^{\prime} C(\tau, T) x^{\tau}(\tau)-\left(x^{\tau}(\tau)\right)^{\prime} B(\tau, T)-A(\tau, T)\right]\right\}
\end{aligned}
$$

and the Kolmogorov backward equation becomes

$$
\left\{\begin{array}{l}
\frac{\partial}{\partial t} p^{\tau}+\left[x^{\prime}\left(F^{\prime}-2 C G G^{\prime}\right)+H^{\prime}-B^{\prime} G G^{\prime}\right] \nabla_{x} p^{\tau}+\operatorname{tr}\left(G^{\prime} \nabla_{x x} p^{\tau} G\right)=0 \\
p^{\tau}(\tau, T, x)=\exp \left[-x^{\prime} C(\tau, T) x-x^{\prime} B(\tau, T)-A(t, \tau)\right]
\end{array}\right.
$$

Following then the steps analogous to (3.22) and (3.23) we end up with the following HJB equation corresponding to (3.24) (we drop the arguments of the functions)

$$
\begin{gathered}
\frac{\partial}{\partial t} W^{\tau}+\min _{u \in \mathbb{R}^{k}}\left\{\left[x^{\prime}\left(F^{\prime}-2 C G G^{\prime}\right)+H^{\prime}-B^{\prime} G G^{\prime}+u^{\prime} G^{\prime}\right]\left(\nabla_{x} W^{\tau}\right)\right. \\
\left.+\frac{1}{2} \operatorname{tr}\left(G^{\prime} \nabla_{x x} W^{\tau} G\right)+\frac{1}{2} u^{\prime} u\right\}=0
\end{gathered}
$$

with minimizer $u(t, x)=-G^{\prime}(t) \nabla_{x} W^{\tau}(t, x)$. This is now the specific form of the HJB equation (3.24) that corresponds to the specific form of (3.26) given by the linear-quadratic regulator problem with state dynamics (3.31) for the state variable $x^{\tau}(t)$ (notice that the presence of the term $H-G G^{\prime} B$ makes this problem of the form of the generalized linearquadratic regulator in Appendix B) and objective function

$$
\begin{aligned}
W^{\tau}(t, T, x)= & \inf _{u \in \mathcal{U}} E_{t, x}\left\{\int_{t}^{\tau} \frac{1}{2} u^{\prime}(s) u(s) d s+\left(x^{\tau}(\tau)\right)^{\prime} C(\tau, T) x^{\tau}(\tau)\right. \\
& \left.+\left(x^{\tau}(\tau)\right)^{\prime} B(\tau, T)\right\}+A(\tau, T)
\end{aligned}
$$

In accordance with Proposition 3.4 we then have that

$$
W^{\tau}(t, T, x)=x^{\prime} C^{\tau}(t, T) x+x^{\prime} B^{\tau}(t, T)+A^{\tau}(t, T)
$$


for $t \leq \tau \leq T$ and, since now in the objective function we have $a(t)=b(t)=c(t)=0$ (for the forward prices we do not have to match the initially observed rates by a suitable choice of $a(t)), C^{\tau}(t, T), B^{\tau}(t, T), A^{\tau}(t, T)$ satisfy

$$
\left\{\begin{array}{c}
\frac{\partial}{\partial t} C^{\tau}(t, T)+\left(F^{\prime}(t)-2 C(t, \tau) G(t) G^{\prime}(t)\right) C^{\tau}(t, T)+C^{\tau}(t, T)(F(t) \\
\left.\quad-2 G(t) G^{\prime}(t) C(t, \tau)\right)-2 C^{\tau}(t, T) G(t) G^{\prime}(t) C^{\tau}(t, T)=0 \\
C^{\tau}(\tau, T)=C(\tau, T) \\
\frac{\partial}{\partial t} B^{\tau}(t, T)+\left[F^{\prime}(t)-2 C(t, \tau) G(t) G^{\prime}(t)-2 C^{\tau}(t, T) G(t) G^{\prime}(t)\right] B^{\tau}(t, T) \\
\quad+2 C^{\tau}(t, T) H(t)-2 C^{\tau}(t, T) G(t) G^{\prime}(t) B(t, \tau)=0 \\
B^{\tau}(\tau, T)=B(\tau, T) \\
\frac{\partial}{\partial t} A^{\tau}(t, T)+H^{\prime}(t) B(t, T)+\operatorname{tr}\left(G^{\prime}(t) C^{\tau}(t, T) G(t)\right) \\
\quad-B^{\prime}(t, T) G(t) G^{\prime}(t) B^{\tau}(t, \tau)-\frac{1}{2} B^{\tau^{\prime}}(t, T) G G^{\prime}(t) B^{\tau}(t, T)=0 \\
A^{\tau}(\tau, T)=A(\tau, T)
\end{array}\right.
$$

which allows to obtain an explicit expression for $W^{\tau}(t, T, x)$.

Note that, contrary to the case of bond prices, here we have the terminal condition $W^{\tau}(\tau, T, x)=x^{\prime} C(\tau, T) x+x^{\prime} B(\tau, T)+A(\tau, T)$. The last equation in (3.37) can be written in integral form as

$$
\begin{aligned}
A^{\tau}(t, T) & =A(\tau, T)+\int_{t}^{\tau} H^{\prime}(s) B(s, T) d s+\int_{t}^{\tau} \operatorname{tr}\left(G^{\prime}(s) C^{\tau}(s, T) G(s)\right) d s \\
& -\int_{t}^{\tau} B^{\tau^{\prime}}(s, T) G(s) G^{\prime}(s) B^{\tau}(s, \tau) d s-\frac{1}{2} \int_{t}^{\tau} B^{\tau^{\prime}}(s, \tau) G(s) G^{\prime}(s) B^{\tau}(s, \tau) d s
\end{aligned}
$$

Finally, as a corollary to Proposition 3.9, we obtain:

Corollary 3.10 For an exponential quadratic term structure, as in the present subsection, the forward price at time $t$ of a bond is given by

$$
E_{t, x}^{Q_{\tau}} p(\tau, T, x)=p^{\tau}(t, T, x)
$$

where

$$
\begin{aligned}
p^{\tau}(t, T, x) & =E_{t, x} \exp \left\{-\left(x^{\tau}(\tau)\right)^{\prime} C(\tau, T) x^{\tau}(\tau)-\left(x^{\tau}(\tau)\right)^{\prime} B(t, \tau)-A(\tau, T)\right\} \\
& =\exp \left\{-\left(x^{\prime} C^{\tau}(t, T) x-\left(x^{\prime} B^{\tau}(t, T)-A^{\tau}(t, T)\right\}\right.\right.
\end{aligned}
$$

\section{Forward measures and bond option pricing}

The results of section 3.3 suggest that a deeper connection exists between the forward prices $p^{\tau}(t, T)$ and the usual forward measure $Q_{\tau}$ which is normally used to compute $(3.16)$. 
In the next section 4.1 we shall now derive this connection for the general case of model (2.1) showing that pricing with the forward measure can be made equivalent to a pricing approach under the standard martingale measure $Q$ by using the forward prices $p^{\tau}(t, T)$. In the following section 4.2 we then specialize this general result to the particular case of an exponential quadratic term structure and for this case in subsection 4.2.1 we present a computable approach for the pricing of a bond derivative.

\subsection{Forward measures and a general pricing formula}

Let

- Let $x(\tau)$ be the value in $\tau$ of the solution to (2.1) with initial condition $x(t)=x$.

- Let $x^{\tau}(\tau)$ be the value in $\tau$ of the solution to (3.19) with initial condition $x^{\tau}(t)=x$

We have

Proposition 4.1 Given $\tau$, the two random variables $x(\tau)$ and $x^{\tau}(\tau)$ have the same distribution, the first under the forward measure $Q_{\tau}$ (with numeraire $p(t, \tau, x)$ ), the second under the standard martingale measure $Q$ (with numeraire $B(t)$ ).

Proof: For the numeraire $p(t, \tau, x)$ we have from (3.5) and (3.6) that, under $Q$,

$$
d p(t, \tau, x)=p(t, \tau, x)\left[r(t) d t-\left[\nabla_{x} W(t, \tau, x)\right]^{\prime} \mathbf{g}(t, x(t)) d w(t)\right]
$$

For the Radon-Nikodym derivative $L(t)=E\left\{\frac{d Q_{\tau}}{d Q} \mid \mathcal{F}_{t}\right\}=\frac{p(t, \tau, x)}{B(t) p(0, \tau, x)}$ one then has

$$
d L(t)=-L(t)\left[\nabla_{x} W(t, \tau, x(t))\right]^{\prime} \mathbf{g}(t, x(t)) d w(t)
$$

It follows that the process $w^{\tau}(t)$ defined by

$$
d w^{\tau}(t)=d w(t)+\left[\nabla_{x} W(t, \tau, x)\right]^{\prime} \mathbf{g}(t, x) d t
$$

is a Wiener process under $Q_{\tau}$.

For $x(t)$ satisfying (2.1) under $Q$ one then has, under $Q_{\tau}$,

$$
d x^{\tau}(t)=\left[\left(\mathbf{f}\left(t, x^{\tau}(t)\right)-\mathbf{g}\left(t, x^{\tau}(t)\right) \mathbf{g}^{\prime}\left(t, x^{\tau}(t)\right) \nabla_{x} W\left(t, \tau, x^{\tau}(t)\right)\right] d t+\mathbf{g}\left(t, x^{\tau}(t)\right) d w(t)\right.
$$

Since the dynamics in (4.4) is identical to those in (3.19) and $x(t)=x$ in both cases, the distribution of $x(\tau)$ under $Q_{\tau}$ and $x^{\tau}(\tau)$ under $Q$ are the same.

Coming now to pricing, we have the following result, which generalizes a scalar result in Pelsser [2000]: 
Proposition 4.2 Given a maturity $\tau$ and an integrable claim $\mathbf{H}(x(\tau))$, its arbitrage free price at $t<\tau$ is

$$
\begin{aligned}
\Pi_{t} & =E_{t, x}\left\{e^{-\int_{t}^{\tau} r(s) d s} \mathbf{H}(x(\tau))\right\} \\
& =p(t, \tau, x) E_{t, x}^{Q_{\tau}}\{\mathbf{H}(x(\tau))\}=e^{-W(t, \tau, x)} E_{t, x}\left\{\mathbf{H}\left(x^{\tau}(\tau)\right)\right\}
\end{aligned}
$$

Proof: The first equality follows from the definition of $Q$ as martingale measure for the numeraire $B(t)$, the second from the definition of the forward measure $Q_{\tau}$ and the third follows from (3.6) and the equality of the distributions of $x(\tau)$ under $Q_{\tau}$ and of $x^{\tau}(\tau)$ under $Q$.

\subsection{The particular case of linear dynamics (exponential quadratic term structure)}

We particularize now the general results of the previous section 4.1 to the exponential quadratic case with linear dynamics for $x(t)$ that (see (4.4) and (2.3)), under $Q_{\tau}$ becomes

$$
d x(t)=\left[\left(F(t)-2 G(t) G^{\prime}(t) C(t, \tau)\right) x(t)+H(t)-G(t) G^{\prime}(t) B(t, \tau)\right] d t+G(t) d w^{\tau}(t)
$$

which is the same as that of $x^{\tau}(t)$ under $Q$ (see (3.31)). Furthermore, the common distribution of $x(\tau)$ under $Q_{\tau}$ and of $x^{\tau}(\tau)$ under $Q$ is Gaussian.

Remark 4.3 An alternative approach to obtain this same (Gaussianity) result in the exponential quadratic case could be to show that from the following equality, that derives from (3.27), namely

$$
\begin{aligned}
E_{t, x}^{Q_{\tau}} & \left\{\exp \left[-x^{\prime}(\tau) C(\tau, T) x(\tau)-x^{\prime}(\tau) B(\tau, T)-A(\tau, T)\right]\right\} \\
& =E_{t, x}\left\{\exp \left[-\left(x^{\tau}(\tau)\right)^{\prime} C(\tau, T) x^{\tau}(\tau)-\left(x^{\tau}(\tau)\right)^{\prime} B(\tau, T)-A(\tau, T)\right]\right\}
\end{aligned}
$$

follows the equality of the two Gaussian distributions, that of $x(\tau)$ under $Q_{\tau}$ and that of $x^{\tau}(\tau)$ under $Q$, given that $x(t)=x^{\tau}(t)=x$.

\subsubsection{Pricing of a bond derivative}

In the context of this subsection, namely of an exponential quadratic term structure we derive now an explicit formula for the pricing of a bond option that is based on (4.5) and on the representation of the factor process $x^{\tau}$ in (3.31). We intend to carry out explicit computations and, to this end, we will need the results in Appendix B where some coefficients are assumed to be constant : we therefore assume from now on that the dynamic coefficients $F, G$ and the coefficients $c, b$ in $r(t, x)$ in (2.3) are constant (in fact, the coefficient $b(t)$ can be a polynomial in $t$, leading to slightly more general structures: this issue, though, will not be pursued here). We also assume that the pair $(F, G)$ is controllable, that is the matrix $G, F G, \ldots F^{n-1} G$ has full column rank. First we have 
Remark 4.4 If $\Phi_{\tau}(\tau, t)$ denotes the fundamental solution of (3.31), we immediately see that, for $\tau>t$ with $\tau<T$ the conditional mean of the Gaussian process $x^{\tau}$ given $x^{\tau}(t)=x$ can be expressed as

$$
E_{t, x} x^{\tau}(\tau)=\Phi_{\tau}(\tau, t) x-\int_{t}^{\tau} \Phi_{\tau}(\tau, s)\left(H(s)-G G^{\prime} B(s, \tau)\right) d s
$$

and its conditional variance by

$$
\begin{aligned}
& E_{t, x}\left[x^{\tau}(\tau)-E_{t, x} x^{\tau}(\tau)\right]\left[\left(x^{\tau}(\tau)\right)^{\prime}-E_{t, x}\left(x^{\tau}(\tau)\right)^{\prime}\right] \\
& \quad=E \int_{t}^{\tau} \Phi_{\tau}(\tau, s) G d\langle w\rangle(s) G^{\prime} \Phi_{\tau}^{\prime}(\tau, s)=\int_{t}^{\tau} \Phi_{\tau}(\tau, s) G G^{\prime} \Phi_{\tau}^{\prime}(\tau, s) d s
\end{aligned}
$$

The integrals (4.8) and (4.9) can be computed numerically, using the explicit representations of $\Phi_{\tau}(\tau, s)$ and $B(s, t)$ (see (B.8) and (B.10) in the Appendix), as well as the alternative representation of the function $\Phi_{\tau}(\tau, s)$ (see (B.5) below). In fact, from (B.8), taking into account that $C(\tau, \tau)=0$, we have

$$
\Phi_{\tau}(\tau, s)=\Phi_{\tau}(s, \tau)^{-1}=\left([I, 0] e^{H(s-\tau)}\left[\begin{array}{l}
I \\
0
\end{array}\right]\right)^{-1}
$$

and from (B.10) and the fact that $B(\tau, \tau)=0$ (assuming, as we said, that $b$ is constant)

$$
\begin{aligned}
B(t, \tau) & =\Phi_{\tau}^{\prime}(t, \tau)^{-1} \int_{\tau}^{t} \Phi_{\tau}^{\prime}(s, \tau) b d s \\
& =\left([I, 0] e^{H^{\prime}(t-\tau)}\left[\begin{array}{l}
I \\
0
\end{array}\right]\right)^{-1} \int_{\tau}^{t}[I, 0] e^{H^{\prime}(s-\tau)}\left[\begin{array}{l}
I \\
0
\end{array}\right] b d s \\
& =\left([I, 0] e^{H^{\prime}(t-\tau)}\left[\begin{array}{l}
I \\
0
\end{array}\right]\right)^{-1}[I, 0]\left(H^{\prime}\right)^{-1}\left(e^{H^{\prime}(t-\tau)}-I\right)\left[\begin{array}{l}
I \\
0
\end{array}\right] b d s
\end{aligned}
$$

The mean (4.8) can now be calculated from (4.10) and (4.11) by numerical computation of a line integral. As for the variance, we can use a numerical integrator using again (4.10).

In view of the previous section, we can express the arbitrage free price of a claim by formula (4.5) where now $x^{\tau}$ is the Gaussian process with mean $\mu$ given by (4.8) and variance $\Sigma$ given by (4.9). In particular, the value of a call option with strike price $K$ and expiration $\tau$ on a bond with maturity $T$ will be,

$$
\begin{aligned}
\Pi_{t} & =e^{-W(t, \tau, x)} E_{t, x} \max \left\{0, p\left(\tau, T, x^{\tau}\right)-K\right\} \\
& =e^{-W(t, \tau, x)} E_{t, x} \max \left\{0, e^{-W(\tau, T, x)}-K\right\}
\end{aligned}
$$

where, in view of (3.11),

$$
\begin{aligned}
& E_{t, x} \max \left\{0, e^{-W(\tau, T, x)}-K\right\} \\
& =\frac{1}{\sqrt{(2 \pi)^{n} \operatorname{det} \Sigma}} \int_{-W(\tau, T, x)>\ln K} e^{-\frac{1}{2}(\xi-\mu)^{\prime} \Sigma^{-1}(\xi-\mu)} e^{-\xi^{\prime} C(\tau, T) \xi-\xi^{\prime} B(\tau, T)-A(\tau, T)} d \xi_{1} \ldots d \xi_{n} \\
& -\frac{K}{\sqrt{(2 \pi)^{n} \operatorname{det} \Sigma}} \int_{-W(\tau, T, x)>\ln K} e^{-\frac{1}{2}(\xi-\mu)^{\prime} \Sigma^{-1}(\xi-\mu)} d \xi_{1} \ldots d \xi_{n}
\end{aligned}
$$


We claim now that $\Sigma$ has full rank (for any given $\tau, T$ ): from (4.9) we see that $\Sigma$ is dependent through $\Phi_{\tau}(\tau, s)$ on the pair $\left(F-2 G G^{\prime} C(t, \tau), G\right)$ (it is, in fact, the controllability gramian of the pair, see Brockett [1970]) and it can be shown that this gramian has full rank if the constant matrix $\left[G, F G, \ldots F^{n-1} G\right]$ has full column rank, a fact that we assume here. We perform next a suitable change of variables remembering that: a) two positive definite matrices can be simultaneously diagonalized by congruence and the transformation can be chosen so that one is the identity; b) if $\Sigma$ is invertible, we can complete the squares. In fact, the quadratic form in the exponent $M(\xi):=\xi^{\prime} C \xi+\xi^{\prime} B+A$ can be written as

$$
M(\xi):=\xi^{\prime} C \xi+\xi^{\prime} B+A=\left(\xi^{\prime}-\nu^{\prime}\right) C(\xi-\nu)-\nu^{\prime} C \nu+A
$$

where we have set

$$
\nu:=-\frac{1}{2} C^{-1} B
$$

To clarify the procedure, observe that, in the two integrals in (4.13), both the exponent of the integrand and the function defining the domain of integration are quadratic functions in $\xi$. Therefore, to compute this kind of integrals, we introduce two quadratic functions $M_{E}$ and $M_{D}$, which will represent the exponent in the integrand and define the domain $D$ of integration respectively. The integrals in (4.13) will then be particular instances of the general derivation obtained in the following Lemma. We have

Lemma 4.5 Let $\xi \in \mathbb{R}^{n}$ and let $A_{E}, B_{E}, C_{E}$ and $A_{D}, B_{D}, C_{D}$ be coefficients of suitable size for the quadratic functions

$$
M_{E}(\xi):=\xi^{\prime} C_{E} \xi+\xi^{\prime} B_{E}+A_{E} \quad M_{D}(\xi):=\xi^{\prime} C_{D} \xi+\xi^{\prime} B_{D}+A_{D}
$$

with $C_{E}$ and $C_{D}$ positive definite and let

$$
D:=\left\{\xi: M_{D}(\xi) \leq-\ln K\right\}
$$

Then

$$
\int_{D} e^{-\frac{1}{2} M_{E}(\xi)} d \xi_{1} \ldots d \xi_{n}=\tilde{\lambda} \int_{\tilde{D}} e^{-\frac{1}{2}|\tilde{\xi}|^{2}} d \tilde{\xi}_{1} \ldots d \tilde{\xi}_{n}
$$

where, with $L^{\prime} L=C_{E}$ and $\nu$ as in (4.15),

$$
\begin{aligned}
\tilde{\xi} & =L(\xi-\nu) \\
\tilde{\lambda} & =e^{\nu^{\prime} C_{E} \nu-A_{E}}|\operatorname{det} L|^{-1} \\
\tilde{D} & =\left\{\tilde{\xi}: M_{D}\left(L^{-1} \tilde{\xi}+\nu\right) \leq-\ln K\right\}
\end{aligned}
$$

Proof: The substitution (4.17) immediately yields $d \tilde{\xi}_{1} \ldots d \tilde{\xi}_{n}=\operatorname{det} L d \xi_{1} \ldots d \xi_{n}$ and

$$
M_{E}(\xi)=\xi^{\prime} C_{E} \xi+\xi^{\prime} B_{E}+A_{E}=\left(\xi^{\prime}-\nu^{\prime}\right) C_{E}(\xi-\nu)-\nu^{\prime} C_{E} \nu+A_{E}=|\tilde{\xi}|^{2}-\nu^{\prime} C_{E} \nu+A_{E}
$$


from which (4.16) follows. Notice that, denoting by $L^{-T}$ the transposed inverse of a matrix $L$,

$$
\begin{aligned}
\tilde{D}= & \left\{\tilde{\xi}: \tilde{\xi}^{\prime} L^{-T} C_{D} L^{-1} \tilde{\xi}+\tilde{\xi}^{\prime} L^{-T}\left(2 C_{D} \nu+B_{D}\right)\right. \\
& \left.+\nu^{\prime} C_{D} \nu+\nu^{\prime} B_{D}+A_{D} \leq-\ln K\right\}
\end{aligned}
$$

We can obviously set

$$
C_{\tilde{D}}:=L^{-T} C_{D} L^{-1} \quad B_{\tilde{D}}:=L^{-T}\left(2 C_{D} \nu+B_{D}\right) \quad A_{\tilde{D}}:=\nu^{\prime} C_{D} \nu+\nu^{\prime} B_{D}+A_{D}+\ln K
$$

and so, with

$$
M_{\tilde{D}}(\xi):=\tilde{\xi}^{\prime} C_{\tilde{D}} \tilde{\xi}+\tilde{\xi}^{\prime} B_{\tilde{D}}+A_{\tilde{D}}
$$

one obtains

$$
\tilde{D}=\left\{\tilde{\xi}: M_{\tilde{D}}(\tilde{\xi}) \leq 0\right\}
$$

Notice that, since $C(t, T)>0$ for $t<T$ (see Remark B.2), the functions $M_{D}(\xi)$ and $M_{\tilde{D}}(\xi)$ are strictly convex and thus the sets $D$ and $\tilde{D}$ are convex.

Thus, in principle, derivative prices can be computed in a standard manner. Nevertheless, these are multiple integrals and thus their actual computation is quite demanding.

In the cases of $n=1$ and $n=2$, though, the formulas can be reduced to calculating the value of single integrals and require therefore a computational effort comparable with that of the Black and Scholes formula.

\subsection{2 $\mathrm{n}=1$ : the CIR case}

It is well known (see Rogers [1995]) that the CIR model is equivalent to a scalar Squared Gaussian model like those presented here. Nevertheless, known computations for such models (see e.g. Pelsser [2000]) involve a double integral for the computation of the mean $\mu$. However, as already mentioned, this is not needed.

In fact, if $n=1$, we can compute (4.13) directly: set, for given $t$ and $T$,

$$
M(\xi):=\xi^{2} C(t, T)+\xi B(t, T)+A(t, T)
$$

Then the domain of integration is $D=\{\xi: M(\xi) \leq-\ln K\}$. Notice that the fact that $C>0$ (see Remark B.2) makes $M(\xi)$ a convex function and thus $D$ is the finite interval delimited by the roots (which we assume real, since otherwise the model has no meaning) of

$$
M(\xi)+\ln K=0
$$


Letting $\xi_{1,2}$ to be these roots with $\xi_{1} \leq \xi_{2}$, the computation for (4.13) reduces to

$$
\begin{aligned}
& \left.E_{t, x}^{Q} \max \left\{0, e^{-W(\tau, T, x(\tau))}-K\right\}=\frac{1}{\sqrt{(2 \pi) \Sigma}} \int_{\xi_{1}}^{\xi_{2}} e^{-\left\{\frac{(\xi-\mu)^{2}}{2 \Sigma}+M(\xi)\right.}\right\} d \xi \\
& \quad-\frac{K}{\sqrt{(2 \pi) \Sigma}} \int_{\xi_{1}}^{\xi_{2}} e^{-\frac{(\xi-\mu)^{2}}{2 \Sigma}} d \xi
\end{aligned}
$$

with $\mu$ and $\Sigma$ computed above. These integrals are standard and the formula is similar to that of Black and Scholes.

\subsubsection{The case $n=2$}

Similarly to the one factor model discussed above, in the case of $n=2$, the formulas, although more complicated, can be reduced to calculating the value of five single integrals and again the computational effort required is comparable to that of Black and Scholes.

The integrals in (4.13) are of the same kind. We show here how to compute the first one. Denote it by $I_{1}$. Set, to this end

$$
C_{E}:=\frac{1}{2} \Sigma^{-1}+C(t, T) \quad B_{E}:=\Sigma^{-1} \mu+B(t, T) \quad A_{E}:=\frac{1}{2} \mu^{\prime} \Sigma^{-1} \mu+A(t, T)
$$

Then

$$
\begin{aligned}
M_{E}(\xi) & :=\frac{1}{2}(\xi-\mu)^{\prime} \Sigma^{-1}(\xi-\mu)+\xi^{\prime} C(\tau, T) \xi+\xi B(\tau, T)-A(\tau, T) \\
& =\xi^{\prime} C_{E} \xi+\xi^{\prime} B_{E}+A_{E}
\end{aligned}
$$

Using the transformations (4.17)-(4.19), we can write:

$$
I_{1}=\frac{1}{2 \pi \sqrt{\operatorname{det} \Sigma}} \tilde{\lambda} \int_{\tilde{D}} e^{-\frac{1}{2}\left(\xi_{1}^{2}+\xi_{2}^{2}\right)} d \xi_{1} d \xi_{2}
$$

Recalling now that

$$
M_{D}(\xi)=W(t, T)+\ln K=\xi^{\prime} C(t, T) \xi+\xi^{\prime} B(t, T)+A(t, T)+\ln K
$$

and using the representation (4.20), (4.21), we obtain

$$
\begin{aligned}
& C_{\tilde{D}}=L^{-T} C(t, T) L^{-1} \\
& B_{\tilde{D}}=L^{-T}(2 C(t, T) \nu+B(t, T)) \\
& A_{\tilde{D}}=\nu^{\prime} C(t, T) \nu+\nu^{\prime} B(t, T)+A(t, T)+\ln K
\end{aligned}
$$

Let now

$$
\left[\begin{array}{ll}
\sigma_{11} & \sigma_{12} \\
\sigma_{21} & \sigma_{22}
\end{array}\right]:=C_{\tilde{D}} \quad\left[\begin{array}{l}
\kappa_{1} \\
\kappa_{2}
\end{array}\right]:=B_{\tilde{D}} \quad \alpha:=A_{\tilde{D}}
$$


Since $C_{\tilde{D}}$ is positive definite, the set $\tilde{D}$ (if it is non empty) is an ellipse. Then, using a standard substitution in (4.20), that is $\tilde{\xi}_{1}=\rho \cos \theta$ and $\tilde{\xi}_{2}=\rho \sin \theta$, we see that the set $\tilde{D}_{\xi_{1} \xi_{2}}:=\left\{\left(\xi_{1}, \xi_{2}\right) ; M_{\tilde{D}}\left(\xi_{1}, \xi_{2}\right) \leq 0\right\}$ is mapped into the set

$$
\tilde{D}_{\rho, \theta}:=\{(\rho, \theta) ; p(\rho, \theta) \leq 0, \rho \geq 0\}
$$

where we have set (using the above substitution):

$$
\begin{aligned}
p(\rho, \theta) & :=\rho^{2}\left(\sigma_{11} \cos ^{2} \theta+\sigma_{22} \sin ^{2} \theta+2 \sigma_{12} \sin \theta \cos \theta\right) \\
& +\rho\left(\kappa_{1} \cos \theta+\kappa_{2} \sin \theta\right)+\alpha
\end{aligned}
$$

As a function of $\rho$, the above is a second degree polynomial with roots $\rho_{1}(\theta), \rho_{2}(\theta)$. Notice that, if $p(\rho, \theta)=0$, also $p(-\rho, \theta+\pi)=0$. Therefore (as long as the discriminant $\Delta$ of (4.23) is non negative), the set of points satisfying (4.23) with $\rho>0$ is non empty. There are two possibilities:

a) The ellipse $\Gamma$ contains the origin, and thus we need to integrate in $\theta$ on $[0,2 \pi)$. Thus, for each $\theta$, only one root of (4.23) is positive. Denoting this root by $\rho_{2}(\theta)$ and setting $k:=\frac{1}{2 \pi \sqrt{\operatorname{det} \Sigma}} \tilde{\lambda}$, we can write $I_{1}$ as

$$
\begin{aligned}
I_{1} & =k \int_{\tilde{D}_{\xi_{1} \xi_{2}}} e^{-\frac{1}{2}\left(\xi_{1}^{2}+\xi_{2}^{2}\right)} d \xi_{1} d \xi_{2}=k \int_{\tilde{D}_{\rho, \theta}} e^{-\frac{1}{2} \rho^{2}} \rho d \rho d \theta=-\left.k \int_{0}^{2 \pi} e^{-\frac{1}{2} \rho^{2}}\right|_{0} ^{\rho_{2}(\theta)} d \theta \\
& =k \int_{0}^{2 \pi}\left[1-e^{-\frac{1}{2} \rho_{2}^{2}(\theta)}\right] d \theta
\end{aligned}
$$

b) The ellipse $\Gamma$ does not contain the origin, and thus both roots $\rho_{1}(\theta), \rho_{2}(\theta)$ have the same sign. Denote therefore by $\Gamma_{\theta}$ the subinterval of $[0,2 \pi)$ where the discriminant $\Delta$ of (4.23) is non negative and $\rho \geq 0$. If we make the convention that $\rho_{1}(\theta) \leq \rho_{2}(\theta)$, we can write $I_{1}$ as:

$$
\begin{aligned}
I_{1} & =k \int_{\tilde{D}_{\xi_{1} \xi_{2}}} e^{-\frac{1}{2}\left(\xi_{1}^{2}+\xi_{2}^{2}\right)} d \xi_{1} d \xi_{2}=k \int_{\tilde{D}_{\rho, \theta}} e^{-\frac{1}{2} \rho^{2}} \rho d \rho d \theta=-\left.k \int_{\Gamma_{\theta}} e^{-\frac{1}{2} \rho^{2}}\right|_{\rho_{1}(\theta)} ^{\rho_{2}(\theta)} d \theta \\
& =k \int_{\Gamma_{\theta}}\left[e^{-\frac{1}{2} \rho_{1}^{2}(\theta)}-e^{-\frac{1}{2} \rho_{2}^{2}(\theta)}\right] d \theta
\end{aligned}
$$

In both cases, this is a simple integral whose value is easily computed numerically. The second integral in (4.13) can be expressed analogously.

To find the extremes of integration (or whether we are in the first or second case), it is sufficient to look at $\alpha$ and at the discriminant

$$
\Delta(\theta)=\left(\kappa_{1} \cos \theta+\kappa_{2} \sin \theta\right)^{2}-4 \alpha\left(\sigma_{11} \cos ^{2} \theta+\sigma_{22} \sin ^{2} \theta+2 \sigma_{12} \sin \theta \cos \theta\right)
$$

In fact, if $\alpha$ is negative, then the origin $(\rho=0)$ is in $\tilde{D}_{\rho, \theta}$ and, in view of the positivity of the matrix $C_{\tilde{D}}$, the quadratic term coefficient is positive and thus $\Delta(\theta)>0$ for all $\theta$. We are thus in the first case. With a bit of care also the case $\alpha=0$ falls within this situation. 
If $\alpha$ is strictly positive, then the origin is not contained in $\tilde{D}_{\rho, \theta}$ and the discriminant will be negative for some values of $\theta$. To find thus the extremes of the interval we need to find the values $\theta$ for which $\Delta(\theta)=0$; to this end, divide $\Delta(\theta)$ by, say, $\cos ^{2} \theta$ to obtain the equation:

$$
\Delta(\theta) \frac{1}{\cos ^{2} \theta}=\left(\kappa_{1}+\kappa_{2} \tan \theta\right)^{2}-4 \alpha\left(\sigma_{11}+\sigma_{22} \tan ^{2} \theta+2 \sigma_{12} \tan \theta\right)=0
$$

which is a quadratic equation in $\tan \theta$.

If the quadratic coefficient of $\tan \theta$ is not zero, this equation will provide four values of $\theta$ in $0,2 \pi$ for which $\Delta$ vanishes. Thus $\Delta$ is positive on two of these intervals. We need to pick the one where both $\rho_{1}$ and $\rho_{2}$ are positive.

If the quadratic coefficient of $\tan \theta$ vanishes, we have a linear equation, and this means that the coefficient of $\sin ^{2} \theta$ in (4.23) is zero as well. Thus two solutions to (4.23) are $\theta=\frac{\pi}{2},-\frac{\pi}{2}$; the other two are derived by the linear equation in $\tan \theta$.

In conclusion, for $n=2$, to compute a call option price at $t$ expiring at $\tau$ on a bond expiring at $T$ we need:

- One line integral to compute $\mu_{t, x}(\tau)$

- One line integral to compute $\Sigma_{t, x}(\tau)$

- One line integral to compute $A(t, T)$.

- Two line integrals to compute the expected value.

All together, we need five line integrals, which are quite fast to compute. The computation of the integrals in (4.13) is now straightforward.

\section{Concluding remarks and possible extensions}

The main purpose of the paper has been to show that for a nonlinear multifactor diffusion type model for the term structure of interest rates, in particular for a linear-quadratic model leading to an exponential quadratic term structure, a feedback approach resulting from a stochastic control methodology provides the same pricing model for bonds and bond derivatives as can be obtained by the traditional martingale approach, but without changing the measure. The basic underlying fact thereby is that, while with the traditional martingale approach we have an implicit change of drift due to a change of measure, in the control approach the drift is changed without changing the measure, but by applying a feedback control. The main advantage of this approach lies, in our view, in the possibility of determining directly the dynamics for the factor process $x^{\tau}$ to compute a forward price (and hence option prices) without having to use the change of measure implied by the 
price process with the new numeraire (which in our case is a more cumbersome exponential quadratic function).

This approach has in particular been worked out for the prices and forward prices of bonds as well as for bond derivatives. This resulted in an efficient approach to compute bond option prices for scalar and two-dimensional linear-quadratic factor models.

Besides its computational effectiveness, the added value of our approach lies also in the various generalizations that can be considered. The change of drift technique, which is implied by the control approach to replace the change of measure from the standard to a more specific martingale measure with a different numeraire, appears to be capable of various extensions (see points i) and ii) below). On the other hand, generalizations of the underlying nonlinear diffusion-type factor model (2.1) to models including jumps appear to lead to difficulties (see points iii) and iv) below).

i) A natural extension that we are currently investigating concerns defaultable bonds and stock options. The main difficulty in the modeling of stock markets seems to be the fact that the present framework leads to the solution of an algebraic Riccati equation; as is well known (see Anderson et al. [1971]), this equation has many solutions, although only one produces a stable dynamics.

ii) An extension to deal with "Swap measures" has been studied in Cogo [2011]: in particular it is shown there that, proceeding analogusly to what was done for forward prices in Section 3.3, one can compute swaption prices as expectations under the standard martingale measure $Q$ by introducing a suitable controlled factor process.

iii) The nonlinear Gaussian model for the factors in (2.1) can be generalized to become a jump-diffusion model or, more generally, a Levy-driven model. While some of the derivations in this paper can be extended without particular problems to the jumpdiffusion setting, constructing an associated control problem via a convex/concave transformation of the prices poses a major problem due to the integral with respect to the jump intensity in the term structure equation. This problem will therefore be investigated elsewhere.

iv) In the linear case (see see (2.3), (3.7), (3.31)) it could make sense from a financial point of view to consider adapted coefficients in the factor dynamics. As mentioned in Remark 3.6, such a model is not Markovian and consequently does not allow to use our PDE-based approach. A nonstandard stochastic control problem formulation would thus be needed and so we leave also this aspect for further investigations.

Acknowledgments: the original thinking about this paper was motivated by some discussion we had many years ago with Professor Tomas Björk: it is a pleasure to acknowledge our debt. We would also like to thank the anonymous referees for some valuable suggestions which helped to considerably improve the paper. 


\section{Appendix}

\section{A Proof of Proposition 3.4}

Proof: It is immediately seen that $u^{*}$ in (3.10) is a stationary point of the function to be minimized in (3.9), but it is just a critical point and, if $b(t) \notin \operatorname{Im} c(t)$, then we cannot complete the squares in (3.9) to eliminate the linear term and use the standard argument of convexity of the quadratic function thus obtained to prove minimality. To see that $u^{*}$ in (3.10) is indeed a minimum in the original problem (3.9), observe first that $W(t, x)$ is bounded from below. In fact, defining

$$
\begin{array}{r}
J(t, x, u):=E_{x}\left\{\int_{t}^{T}\left(x^{\prime}(s) c(s) x(s)+x^{\prime}(s) b(s)+a(s)+\frac{1}{2} u^{\prime}(s) u(s)\right) d s\right. \\
\left.+x^{\prime}(T) C x(T)+x^{\prime}(T) B+A\right\}
\end{array}
$$

we have

$$
\begin{array}{ll}
J_{0}(t, T, x, u) & :=E_{t, x}\left\{\int_{t}^{T}\left[\frac{1}{2} u^{\prime}(s) u(s)+x^{\prime}(s) b(s)\right] d s\right\} \\
(\text { A.2) } & =J(t, T, x, u)-\int_{t}^{T}\left[x^{\prime}(s) c(s) x(s)+a(s)\right] d s \leq J(t, x, u ; T)-\int_{t}^{T} a(s) d s
\end{array}
$$

for any $u(t)$. We can now express $J_{0}(t, T, x, u)$ as the sum of a non negative integral depending on $u$ and a deterministic term independent of the control. In fact, denoting by $\Phi(s, t)$ the fundamental solution associated with (3.7), we get for $s>t$

$$
x(s)=\Phi(s, t) x(t)+\int_{t}^{s} \Phi(s, \tau)[(G(\tau) u(\tau)+H(\tau)) d \tau+G(\tau) d w(\tau)]
$$

Since $x(t)$ is measurable with respect to $\mathcal{F}_{t}$, it can be brought outside the expected value and there is therefore no loss of generality in assuming $x=x(t)=0$. Thus substituting (A.3) into the expression (A.2) (and using the fact that $x^{\prime}(s) b(s)=b^{\prime}(s) x(s)$ ), one obtains

$$
\begin{aligned}
& J_{0}(t, T, x, u)=E_{t, x}\left\{\int_{t}^{T}\left[\frac{1}{2} u^{\prime}(s) u(s)+b^{\prime}(s) x(s)\right] d s\right\} \\
& =E_{t, x}\left\{\int_{t}^{T}\left[\frac{1}{2} u^{\prime}(s) u(s)+b^{\prime}(s) \int_{t}^{s} \Phi(s, \tau)[(G(\tau) u(\tau)+H(\tau)) d \tau+G(\tau) d w(\tau)]\right] d s\right\} \\
& =E_{t, x}\left\{\int_{t}^{T} \frac{1}{2} u^{\prime}(s) u(s) d s+\int_{t}^{T} \int_{t}^{s} b^{\prime}(s) \Phi(s, \tau)[G(\tau) u(\tau)+H(\tau)] d \tau d s\right\} \\
& =E_{t, x}\left\{\int_{t}^{T} \frac{1}{2} u^{\prime}(s) u(s) d s+\int_{t}^{T} \int_{\tau}^{T} b^{\prime}(s) \Phi(s, \tau) d s G(\tau) u(\tau) d \tau+\int_{t}^{T} \int_{\tau}^{T} b^{\prime}(s) \Phi(s, \tau) d s H(\tau) d \tau\right\}
\end{aligned}
$$


Setting $\psi^{\prime}(\tau):=\int_{\tau}^{T} b^{\prime}(s) \Phi(s, \tau) d s$, we can complete the squares and write:

$$
\begin{aligned}
J_{0}(t, T, x, u)= & E_{t, x}\left\{\int_{t}^{T} \frac{1}{2} u^{\prime}(s) u(s) d s+\int_{t}^{T} \psi^{\prime}(s) G(s) u(s) d s+\int_{t}^{T} \psi^{\prime}(s) H(s) d s\right\} \\
= & E_{t, x}\left\{\int_{t}^{T}\left[u^{\prime}(s), 1\right]\left[\begin{array}{cc}
\frac{1}{2} I_{k} & \frac{1}{2} G^{\prime}(s) \psi(s) \\
\frac{1}{2} \psi^{\prime}(s) G(s) & \frac{1}{2} \psi^{\prime}(s) G^{\prime}(s) G(s) \psi(s)
\end{array}\right]\left[\begin{array}{c}
u(s) \\
1
\end{array}\right] d s\right\} \\
& -\int_{t}^{T} \frac{1}{2} \psi^{\prime}(s) G^{\prime}(s) G(s) \psi(s) d s+\int_{t}^{T} \psi^{\prime}(s) H(s) d s
\end{aligned}
$$

The matrix in the first integral is non negative definite and the second and third integrals

do not depend on $u$. Thus $J_{0}(t, T, x, u)$ has a minimum. Since $J_{0}(t, T, x, u)+\int_{t}^{T} a(s) d s$ is bounding $J(t, T, x, u)$ from below, also $J(t, T, x, u)$ has a minimum given by $W(t, x)$. Given the quadratic nature of the function to be minimized, it has only one critical point which thus coincides with the minimum.

Making now the usual Ansatz by putting as in (3.11) $W(t, x)=x^{\prime} C(t) x+x^{\prime} B(t)+A(t)$, we obtain

$$
\begin{cases}\frac{\partial W}{\partial t}(t, x) & =x^{\prime} \frac{d}{d t} C(t) x+x^{\prime} \frac{d}{d t} B(t)+\frac{d}{d t} A(t) \\ \nabla_{x} W(t, x) & =2 x^{\prime} C(t)+B(t) \\ \nabla_{x x} W(t, x) & =2 C(t)\end{cases}
$$

and (see $(3.10)) u^{*}=-G^{\prime}(t)(2 C(t) x+B(t))$. Inserting these expressions into the HJB equation (3.9) one has

$$
\begin{aligned}
& x^{\prime}\left[\frac{\partial}{\partial t} C(t)+c(t)+2 C(t) G(t) G^{\prime}(t) C(t)+2 C(t) F(t)-4 C(t) G(t) G^{\prime}(t) C(t)\right] x \\
& +x\left[\frac{\partial}{\partial t} B(t)+b(t)+C(t) G(t) G^{\prime}(t) B(t)+C(t) G(t) G^{\prime}(t) B(t)+F^{\prime}(t) B(t)\right. \\
& \left.-2 C(t) G(t) G^{\prime}(t) B(t)+2 C(t) H(t)-2 C(t) G(t) G^{\prime}(t) B(t)\right] \\
& +\left[\frac{\partial}{\partial t} A(t)+a(t)+\frac{1}{2} B^{\prime}(t) G(t) G^{\prime} B(t)+B^{\prime}(t) H(t)-B^{\prime}(t) G G^{\prime} B(t)+\operatorname{tr}\left(G C(t) G^{\prime}\right)\right]=0
\end{aligned}
$$

Simplifying and imposing that the equation has to hold for all $x \in \mathbb{R}^{n}$, we obtain the result. Uniqueness follows from that of the solutions to the above differential equations (see the following appendix B for details).

The terminal conditions follow immediately from the terminal condition in (3.9).

\section{B Differential Riccati equation and closed loop dy- namics: computation of the solutions}

The computation of bond prices and forward prices as well as of interest rates becomes definitely simple provided we have a solution to the equations (3.12) and, analogously to 
(3.37). We provide now an explicit solution $C(t, T)$ to $(3.12)$ provided we have a solution to two time invariant equations of which the solution can be easily computed numerically. This will suffice if $B \equiv 0$. Otherwise, a fundamental solution of the second equation in (3.12) is needed. It turns out that also this can be computed explicitly. We follow Anderson et al. [1971] for this approach (see also Yong et al. [1999]).

Since, for each $T$, those in (3.12) are ordinary differential equations, to simplify the notation, in what follows we will drop the explicit dependence on this variable $T$ as well as the dependence on $t$ where possible.

We have the following general result that leads to a solution of the first equation in $(3.12)$.

Theorem B.1 Let $C(t)$ be a solution to

$$
\left\{\begin{array}{l}
\frac{d C(t)}{d t}+C(t) F(t)+F^{\prime}(t) C(t)-C(t) G(t) N^{-1}(t) G^{\prime}(t) C(t)+c(t)=0 \\
C\left(t_{1}\right)=C_{1}
\end{array}\right.
$$

where $C_{1}$ and $c(t)$ are symmetric non negative definite and $N(t)$ is positive definite on the interval $\left[t_{0}, t_{1}\right]$. Then a solution $C(t)$ to (B.1) always exists between $t_{0}$ and $t_{1}$ and it can be expressed as

$$
C(t)=Y(t) X(t)^{-1}
$$

where $X$ and $Y$ satisfy the following linear differential equation:

$$
\frac{d}{d t}\left[\begin{array}{l}
X \\
Y
\end{array}\right]=\left[\begin{array}{cc}
F & -G N^{-1} G^{\prime} \\
-c & -F^{\prime}
\end{array}\right]\left[\begin{array}{l}
X \\
Y
\end{array}\right] \quad\left[\begin{array}{c}
X\left(t_{1}\right) \\
Y\left(t_{1}\right)
\end{array}\right]=\left[\begin{array}{c}
I \\
C_{1}
\end{array}\right]
$$

Moreover, if $\Phi(t, s)$ denotes the fundamental solution associated with

$$
\frac{d x(t)}{d t}=\left[F(t)-G(t) N^{-1}(t) G^{\prime}(t) C(t)\right] x(t)
$$

then $X$ and $Y$ admit the following interpretation

$$
X(t)=\Phi\left(t, t_{1}\right)
$$

and (in view of (B.2))

$$
Y(t)=C(t) \Phi\left(t, t_{1}\right)
$$

Proof: Let $\left[\begin{array}{l}X(t) \\ Y(t)\end{array}\right]$ be a solution to (B.3). Suppose first $X(t)$ is invertible. Then, we can write (dropping the dependence on $t$ ), in view of (B.3),

$$
\begin{aligned}
\frac{d}{d t} C & =\frac{d}{d t}\left[Y X^{-1}\right]=\left(\frac{d}{d t} Y\right) X^{-1}-Y X^{-1} \frac{d}{d t}(X) X^{-1} \\
& =-c X X^{-1}-F^{\prime} Y X^{-1}-Y X^{-1} F X X^{-1}+Y X^{-1} G N^{-1} G^{\prime} Y X^{-1} \\
& =-c-F^{\prime} C-C F+C G N^{-1} G^{\prime} C
\end{aligned}
$$


as wanted according to (B.1). Conversely, if $C(t)$ exists in $\left(t_{0}, t_{1}\right)$, the differential equation (B.4) has the fundamental solution $\Phi(t, s)$, which is defined for $t_{0} \leq t, s \leq t_{1}$ and thus it is invertible on that interval. Moreover,

$$
\begin{aligned}
\frac{d}{d t} \Phi\left(t, t_{1}\right) & =\left[F(t)-G(t) N^{-1}(t) G^{\prime}(t) C(t)\right] \Phi\left(t, t_{1}\right) \\
& =F(t) \Phi\left(t, t_{1}\right)-G(t) N^{-1}(t) G^{\prime}(t) C(t) \Phi\left(t, t_{1}\right)
\end{aligned}
$$

and

$$
\begin{gathered}
\frac{d}{d t} C(t) \Phi\left(t, t_{1}\right)=\frac{d C(t)}{d t} \Phi\left(t, t_{1}\right)+C(t) \frac{d}{d t} \Phi\left(t, t_{1}\right) \\
\left.=-\left[C(t) F(t)+F^{\prime}(t) C(t)-C(t) G(t) N^{-1}(t) G^{\prime}(t) C(t)+c(t)\right] \Phi\left(t, t_{1}\right)\right] \\
\quad+C(t) F(t) \Phi\left(t, t_{1}\right)-C(t) G(t) N^{-1}(t) G^{\prime}(t) C(t) \Phi\left(t, t_{1}\right) \\
=-c(t) \Phi\left(t, t_{1}\right)-F^{\prime}(t) C(t) \Phi\left(t, t_{1}\right)
\end{gathered}
$$

Since $\Phi\left(t_{1}, t_{1}\right)=I$, setting $C\left(t_{1}\right)=C_{1}$, we get the second row in (B.3) by putting $Y(t)=C(t) \Phi\left(t, t_{1}\right)$. The conclusion then follows by the uniqueness of the solution to (B.6).

Notice that, if $F, G, N, c$ are constant, setting $H=\left[\begin{array}{cc}F & -G N^{-1} G^{\prime} \\ -c & -F^{\prime}\end{array}\right]$ to be the Hamiltonian matrix in (B.3), we obtain the explicit representation

$$
\Phi\left(t, t_{1}\right)=X(t)=[I, 0] e^{H\left(t-t_{1}\right)}\left[\begin{array}{c}
X\left(t_{1}\right) \\
Y\left(t_{1}\right)
\end{array}\right]
$$

Remark B.2 Suppose $C_{1}$ and $c(t)$ are symmetric non negative definite and $N(t)$ is positive definite on the interval $\left[t_{0}, t_{1}\right]$. Then $C(t) \geq 0$ for $t<t_{1}$ (see e.g. Fleming et al. [1975]). If moreover $F, G$ is controllable, then $C(t)>0$ for $t<t_{1}$.

Concerning the solution of the second equation in (3.12) we have now (recall that in our case we have $N=\frac{1}{2}$ )

Corollary B.3 Let $B(t)$ be the solution to

$$
\left\{\begin{array}{l}
\frac{d B(t)}{d t}+\left[F^{\prime}(t)-C(t) G(t) N^{-1}(t) G^{\prime}(t)\right] B(t)+b(t)=0 \\
B\left(t_{1}\right)=B_{1}
\end{array}\right.
$$

and let $\Phi(t, s)$ be the fundamental solution for the system (B.9). Then $B(t)$ can be written as

$$
B(t)=\Phi^{\prime}\left(t_{1}, t\right) B_{1}+\int_{t_{1}}^{t} \Phi^{\prime}(s, t) b(s) d s=X^{\prime}(t)^{-1} B_{1}+\int_{t_{1}}^{t}\left[X^{-1}(t)\right]^{\prime} X^{\prime}(s) b(s) d s
$$


Proof: Since, in view of (B.4),

$$
\frac{d}{d t} \Phi(t, s)=\left[F(t)-G(t) N^{-1}(t) G^{\prime}(t) C(t)\right] \Phi(t, s)
$$

and $\Phi(t, s)^{-1}=\Phi(s, t)$, we get that

$$
\frac{d}{d s} \Phi^{\prime}(t, s)=\frac{d}{d s}\left[\Phi(s, t)^{-1}\right]^{\prime}=\left[F^{\prime}(s)-C(s) G(t) N^{-1}(s) G^{\prime}(t)\right] \Phi^{\prime}(t, s)
$$

and thus the result.

Again, if $F, G, N, c$ are constant, using the representation (B.8), we easily see that, if also $b$ is a constant vector (or even a polynomial vector) the integral (B.10) can be computed explicitly.

\section{References}

B.D.O. Anderson, J.B. Moore, 1971. Linear Optimal Control, Prentice-Hall.

T. Björk, 2004. Arbitrage Theory in Continuous Time, Oxford University Press.

R. Brockett, 1970, Finite dimensional linear systems. John Wiley and sons, Inc.

R.Cogo, 2011. Stochastic control and the pricing of interest rate derivatives. Master's thesis, University of Padova.

W.H. Fleming, 1982. Logarithmic transformations and stochastic control, in Advances in Filtering and Optimal Stochastic Control, Lecture Notes in Control and Information Sciences, vol 42, Springer, Berlin.

W.H. Fleming and R.W. Rishel, 1975. Deterministic and Stochastic Optimal Control, Springer.

D. Filipovic, 2002. Separable Term Structures and the Maximal Degree Problem, Mathematical Finance 12/4, pp. 341-349.

A. Friedman, Stochastic Differential Equations and Applications, Vol. 1, Academic Press, 1975.

Y.Hu and X.Y. Zhou, 2005. Constrained stochastic LQ control with random coefficients, and application to mean-variance portfolio selection, SIAM Journal on Control and Optimization, 44, pp. 444-466.

R.C. Merton, 1971. Optimal consumption and Portfolio Rules in a Continuous Time model, Journal of Economic Theory, 3, pp. 373-413. 
B. Øksendal, 1998. Stochastic Differential Equations, Springer-Universitext.

A. Pelsser, 2000. Efficient Methods for Valuing Interest rates Derivative, Spinger.

L. C. G. Rogers, 1995. Which model for term-structure of interest rates should one use?, In : Mathematical Finance (M.H.A. Davis et al. eds.), Springer- Verlag, New York, pp. 93-115.

J. Yong and X. Zhou, 1999. Stochastic Controls: Hamiltonian Systems and HJB Equations, Springer, New York. 